\title{
Analysis of the audiological characteristics and comorbidity in patients with chronic tinnitus
}

\author{
Silva Pavaci, ${ }^{1,2}$ Federica Tortorella, ${ }^{1,2}$ Alessandra Barbara Fioretti, ${ }^{3}$ Anna Maria Angelone, ${ }^{4}$ \\ Lino Di Rienzo Businco, ${ }^{2}$ Maria Lauriello, ${ }^{1}$ Alberto Eibensteinn \\ ${ }^{1}$ Department of Applied Clinical and Biotechnological Sciences, University of l'Aquila, L'Aquila; \\ ${ }^{2}$ Minimally Invasive ENT Surgery Unit, San Carlo di Nancy Hospital, Roma; ${ }^{3}$ Tinnitus Center, European \\ Hospital, Roma; ${ }^{4}$ Department of Life, Health and Environmental Sciences, University of l'Aquila, Italy
}

\begin{abstract}
Tinnitus is defined as perception of a sound without sound stimulation. This study aims to investigate the correlation between chronic tinnitus and the most significant clinical comorbidities and pharmacological treatments. We recruited 130 consecutive outpatients with a tinnitus for least from three months and 100 subjects without tinnitus. All patients had a full medical and audiological evaluation and all filled in Tinnitus Handicap Inventory questionnaire and Khalfa's Hyperacusis questionnaire. We also analyzed the qualitative variables: audiometry exam, tinnitus characteristics and psychometric questionnaires. Univariate logistic regression was performed to evaluate the associations between the presence of tinnitus and the presence of comorbidities and drug intake. The statistical analysis provided the following results in the group of patients with tinnitus. We obtained an Odds Ratio statistically significant for the following categories taken into consideration: the presence of anxiety and depression, neurological diseases, headache, temporomandibular joint (TMJ) disorders, intake of levothyroxine and proton-pump inhibitor. In this study, we tried to evaluate the audiological characteristics in the subjects affected by chronic tinnitus in order to find a possible correlation with the comorbidities and any drugs intake. We found a statistically significant correlation between tinnitus and comorbidities like anxiety, depression, TMJ disorders, dysthyroidism, headache and levothyroxine and PPI intake.
\end{abstract}

\footnotetext{
Correspondence: Silva Pavaci, Minimally Invasive ENT Surgery Unit, San Carlo di Nancy Hospital, via Agrigento 6, 00161 Roma, Italy. E-mail: silpavaci@yahoo.it

Key words: Tinnitus; comorbidity; drugs medication; levothyroxine; proton-pump inhibitor.

Received for publication: 28 July 2019.

Revision received: 7 October 2019.

Accepted for publication: 31 October 2019.

This work is licensed under a Creative Commons Attribution NonCommercial 4.0 License (CC BY-NC 4.0).

${ }^{\circ}$ Copyright: the Author(s), 2019

Licensee PAGEPress, Italy

Audiology Research 2019:9:231

doi:10.4081/audiores.2019.231
}

\section{Introduction}

Tinnitus is defined by the American National Standards Institute ${ }^{1}$ as the perception of a sound without sound stimulation. The definition of conscious sound experience in the head was proposed by the Committee on Hearing, Bioacoustics and Biomechanics. $^{2}$

Tinnitus can occur in association with otological diseases (like otosclerosis, Ménière's disease and vestibular schwannoma) as well as other metabolic abnormalities and psychiatric disturbances (like anxiety and depression). Specifically, sensorineural hearing loss, obesity, smoking, alcohol abuse, previous head trauma, hypertension, sleep disorders, and ototoxic drugs (such as salicylates) are possible risk factors for sensorineural type tinnitus. Somatosensory tinnitus is attributed to the frequent co-existence of tinnitus and TMJ disorders. ${ }^{3}$

In the United States the prevalence of tinnitus increases with age, standing at $31.4 \%$ in the age group between 60 and 69 years. The incidence of tinnitus is higher in males, non-Hispanic white, with a body mass index $\geq 30 \mathrm{~kg} / \mathrm{m}^{2}$, diagnosed with hypertension or diabetes mellitus or dyslipidemia or anxiety disorder. ${ }^{4}$

Recent American guidelines recommend during the initial assessment of a patient with presumed primary tinnitus, to perform a medical history and physical examination to identify conditions that, if promptly identified and managed, could improve the subjective tinnitus annoyance. The evaluation of unilateral tinnitus considering first the suspicion of vestibular schwannoma or vascular tumor firstly should be completed with the otological and audiological evaluation, and if it's also indicated with the imaging. The potentially harmful effects of occupational or recreational exposure to noise can lead to an acoustic shock which must be promptly evaluated from a complete audiological point of view. ${ }^{5}$

Moreover, a number of recent studies highlighted a correlation between tinnitus and various systemic and organ diseases.

For instance, it was observed that cardiovascular diseases and vascular lesions can cause tinnitus, whereas the prompt treatment setting can alleviate the symptoms of tinnitus. ${ }^{6}$

Also, pathologies like anxiety and depression are common in patients with tinnitus. The association between major depression and tinnitus was reported in $48 \%-60 \%$ of cases $^{7}$ and the severity of depression and anxiety is related to the severity of tinnitus. ${ }^{8}$

A recent study emphasizes the influence of the type and side of headache and the characteristics of tinnitus demonstrating greater impairment in patients with left and bilateral headache and in patients with migraine or cluster headache. ${ }^{9}$

A 2013 study showed that there is a high risk of developing hearing loss in individuals suffering from gastroesophageal reflux 
especially in the women; while this has not been demonstrated in case use of pump proton inhibitors. ${ }^{10}$

Tinnitus can also be evoked or modulated by the inputs of somatosensory, somatomotory and visual-motor systems in some individuals. This means that the volume and tone of the tinnitus could be changed immediately, even if temporarily, by different stimuli. ${ }^{11}$

Therefore, this study aims to investigate the correlation between the audiological characteristics of chronic tinnitus and the most significant clinical comorbidities and their pharmacological treatments.

In detail we wanted to assess the audiological characteristics of tinnitus in our group of patients with comorbidities to understand if in this group of subjects, it is possible to find a precise correlation in order to intervene in time to reduce the sensation of tinnitus or improve it. In addition, by evaluating the nature of tinnitus in all patients treated with drugs intake we wanted to evaluate the risk factors of ototoxicity and when preventive measures can be implemented to prevent the onset of tinnitus.

\section{Materials and Methods}

\section{Participants}

We recruited 130 consecutive outpatients with a primary complaint of tinnitus for at least three months. All patients filled in the complete Italian version of the Khalfa's hyperacusis questionnaire (HQ) and the tinnitus handicap inventory (THI) in a self-administered way. An accurate anamnesis was performed. Patients also underwent an ENT clinical examination with otoscopy, rhinoscopy and pharingoscopy. The audiological examination was carried out in an audiological cabin, using an audiometer (Madsen Itera II, GN Otometrics) and included tonal audiometry, pitch and loudness tinnitus matching, and determination of Uncomfortable Loudness Levels (ULLs). Normal hearing was defined by threshold of $<25 \mathrm{~dB} \mathrm{HL}$ in all frequencies tested between $125 \mathrm{~Hz}$ and 8000 $\mathrm{Hz}$.

A homogeneous control group of 100 subjects was collected, which did not show tinnitus and hearing loss.

In our study, the selected participants of the tinnitus group had normal hearing or a mild Sensorineural hearing loss (SNHL) only at high frequencies $(6000$ and $8000 \mathrm{~Hz})$.

The exclusion criteria were the presence of pulsatile tinnitus, the presence of tinnitus for less than three months (acute tinnitus), schizophrenic patients, children under 18 years and subjects that do not understand Italian language.

Informed consent was obtained from each participant before the ENT visit and the collection of clinical data. Each patient gave approval for the processing of personal data anonymously for a possible study.

Considering that our study is observational, carried out using questionnaires and data from non-invasive routine exams, such as audiometry, no formal medical approval by the local medical ethics committee was requested.

\section{Tonal audiometric examination}

The tonal audiometric examination was performed by means of monaural stimulation in the headphones, for the detection of the air threshold (at frequencies 125, 250, 500, 1000, 2000, 3000, 4000, 6000 and $8000 \mathrm{~Hz}$ ), and with mastoid vibrator, for the bone threshold relief (at frequencies $250-4000 \mathrm{~Hz}$ ). The entity of hearing loss was defined, established on the recommendations of the
Committee on Hearing and Equilibrium of the American Academy of Otolaryngology, Head and Neck Surgery of 1995, and based on the Pure Tone Average. ${ }^{12}$

\section{Uncomfortable loudness levels}

The ULLs were determined by a pre-established methodological process. In our study the frequencies tested were $0.25,0.5,1,2,4$ and $8 \mathrm{kHz}$.

All ULLs were measured by providing precise instructions to patients, as indicated by the British Society of Audiology. ${ }^{13}$

\section{Distortion product otoacoustic emissions}

Distortion product otoacoustic emissions (DPOAEs) were measured in both ears and the results were reported as pass/refer.

\section{Khalfa's hyperacusis questionnaire, the tinnitus handi- cap inventory and anamnestic collection}

Since there are no objective tests for the evaluation tinnitus, clinicians must rely on questionnaires to assess the severity of the disorder. $^{5}$

The most commonly tool used for this purpose is the THI, which defines the perceived impact of symptoms on the patient's quality of life and currently has a standardized Italian version. ${ }^{14}$

In a previous study we validated the Italian version of the HQ and we found a cut off score of 16 as indicative of the presence of hyperacusis. ${ }^{16}$

The anamnestic collection was carried out to obtain information about comorbidities and drugs intake.

\section{Statistical analysis}

A descriptive analysis was performed by calculating the relative frequencies for dichotomous variables: gender, drugs (antihypertensive, diuretic, cortisone, levothyroxine, hypoglycemic agents, proton-pump inhibitors (PPI) and antidepressants) and associated diseases such as anxiety, depression, neurological diseases, hypertension, cardiovascular diseases, diabetes, headache and TMJ disorders. The qualitative variables considered were audiometry exam, tinnitus pitch and loudness trend, tinnitus affected side, and patient description of tinnitus. The mean and the standard deviation for the continuous variables (Age, Total THI, Total HQ) were also evaluated.

$\chi^{2}$ test was used in frequency distributions for intensity variables in both the right ear and the left ear.

In order to examine the possible associations between the tinnitus and comorbidities or drug intake (dichotomous variables), a univariate logistic regression was performed, evaluating the Odds Ratio (OR) and the respective confidence intervals. A p-value of less than 0.05 was considered significant.

The data were analyzed using the Stata 12 software (Stata Corp LP, College Station, TX, USA).

\section{Results}

The statistical analysis provided the following results in the group of patients with tinnitus: the sample consisted of 75 males $(57.7 \%)$ and 55 females (42.3\%), age range 22-81 years, (mean=53 years), the control group represented by 54 male and 46 female, age range $28-62$ years (mean $=46$ years). The two groups did not present statistically significant differences in age and sex distribution.

Table 1 shows the results for the individual variables: gender demographics (male/female distribution) and audiological characteristics [presence of hearing loss, values of ULLs and 
Distortion product otoacoustic emissions (DPOAEs), measured a level of both ears]. We have also examined the distribution of the values obtained at each individual side of the ULLs and DPOAEs.

We analyzed the distribution and the scores obtained by psychometric questionnaires: the average score of the THI questionnaire obtained was of 31.65 (29 for male and 35.4 for female subjects). A logistic regression for sex was performed and showed a significant difference between the two sexes regarding the variable of the total THI ( $p$-value $<0.05$ ). HQ's scores were evaluated in patients with tinnitus to consider the presence of hyperacusis. In our sample, $28.5 \%$ of patients $(n=37)$ reported a value below 16 and $71.5 \%$ of patients $(n=93)$ reported a value greater than 16. Male subjects obtained an average total score of the HQ score of 11.3 and females presented an average value of 14.6. The logistic regression performed showed a statistical significance based on gender ( $p$-value $<0.05$ ).

We also evaluated the frequency and intensity distribution for both sides in all patients.

These two variables for every single side have been crossed and evaluated with the $\chi^{2}$ test, showing a significance for the left side of $\mathrm{p}=0.021$. The right side did not show any statistical significance $(p=0.9)$. These results indicate that the left side was more affected but above all the most affected frequencies were higher than 6000 $\mathrm{Hz}$ with an average intensity of $41.3 \mathrm{~dB}$. Also, DPOAEs were-more affected on the left side, ULLs did not show significant relevance ( $p$-value $>0.05$ ). A model was developed to assess comorbidities with tinnitus logistically; the obtained ORs were statistically significant for the following categories taken into consideration: the presence of neurological diseases, depression, anxiety, TMJ disorders, headache, dysthyroidism, intake of levothyroxine and proton-pump inhibitors (Tables 2 and 3).

\section{Discussion}

In this study many patients affected by tinnitus also presented an association with comorbidities and drugs intake. Specifically, we found a statistically significative correlation with TMJ disorders, anxiety, depression, dysthyroidism, headaches and with the assumption of levothyroxine and proton-pump inhibitors. The impairment was evaluated with an accurate audiological examination and successively confirmed through subjective questionnaires on tinnitus annoyance and eventually on hyperacusis.

Our findings are in line with earlier studies, recent article Kim et. al identified many risk factors associated with tinnitus: being female smoking, reduced number of hours of sleep ( $\leq 6$ hours), stress, rheumatoid arthritis, asthma, depression, thyroid diseases, tympanic membrane abnormalities, unilateral or bilateral hearing loss, exposure to earphone noise, exposure to noise at work, exposure to noise outside the workplace and short exposure to noise and hyperlipidaemia. ${ }^{17}$

The increased incidence of tinnitus in patients with TMJ disorders compared to the general population (32\% according to $\mathrm{Bush}^{18}$ ) indicates a correlation between tinnitus and TMJ disorders, such as to represent the cause of $7 \%$ of all tinnitus.

Some studies have shown that somatosensory tinnitus could be due to somatosensory input afferent from the trigeminal and dorsal ganglia that cause changes in the frequency and synchrony of discharge to the dorsal cochlear nuclei, which could trigger somatic tinnitus. ${ }^{19}$

Table 1. Demographic data and audiological evaluation.

\begin{tabular}{lcc} 
Clinical features & $\begin{array}{c}\text { Patients with tinnitus } \\
(\mathrm{n}=130)\end{array}$ & $\begin{array}{c}\text { Control group } \\
(\mathrm{n}=100)\end{array}$ \\
Age(years) (mean \pm SD) & $53.1 \pm 13.97$ & $46.16 \pm 12.95$ \\
Gender (male)(Freq. (\%)) & $75(57.69)$ & $54(54)$ \\
\hline Audiometric examination (normal) & $52(40)$ & $7(93)$ \\
ULLs (right-nomal) & $95(73)$ & $2(98)$ \\
\hline ULLs (left-normal) & $96(75)$ & $3(97)$ \\
DPOAEs (right-pass) & $42(32.3)$ & $4(96)$ \\
\hline DPOAEs (left-pass) & $40(29.2)$ & $7(93)$ \\
\hline
\end{tabular}

Table 2. Pharmacological therapies and associated diseases of the compared samples.

\begin{tabular}{|c|c|c|c|}
\hline & $\begin{array}{l}\text { Patients with tinnitus } \\
\qquad(\mathrm{n}=130)\end{array}$ & $\begin{array}{l}\text { Control group } \\
(n=100)\end{array}$ & P-value \\
\hline Gender (male) (Freq.(\%)) & $75(57.69)$ & 54 & n.s \\
\hline $\begin{array}{l}\text { Drugs } \\
\text { Antihypertensive } \\
\text { Diuretics } \\
\text { Cortisone } \\
\text { Levothyroxine } \\
\text { Hypoglycemic drugs } \\
\text { Proton-pump inhibitors } \\
\text { Antidepressants }\end{array}$ & $\begin{array}{c}73(56.15) \\
32(24.62) \\
14(10.77) \\
5(3.85) \\
27(20.77) \\
12(9.23) \\
20(15.38) \\
14(10.77)\end{array}$ & $\begin{array}{c}51 \\
23 \\
10 \\
3 \\
10 \\
7 \\
7 \\
5\end{array}$ & $\begin{array}{l}\text { n.s } \\
\text { n.s } \\
\text { n.s } \\
\text { n.s } \\
0.08 \\
\text { n.s } \\
0.02 \\
\text { n.s }\end{array}$ \\
\hline $\begin{array}{l}\text { Associated diseases } \\
\text { TMJ Disorders } \\
\text { Headache } \\
\text { Neurological disorders } \\
\text { Depression } \\
\text { Anxiety } \\
\text { Cardiovascular diseases } \\
\text { Hypertension } \\
\text { Diabetes } \\
\text { Dyslipidemia } \\
\text { Dysthyroidism } \\
\end{array}$ & $\begin{array}{c}63(48.46) \\
59(45.38) \\
12(9.23) \\
35(26.92) \\
71(54.62) \\
14(10.77) \\
38(29.23) \\
14(10.77) \\
9(6.92) \\
34(26.15) \\
\end{array}$ & $\begin{array}{c}33 \\
31 \\
2 \\
6 \\
36 \\
17 \\
21 \\
7 \\
7 \\
12 \\
\end{array}$ & $\begin{array}{c}0.001 \\
0.001 \\
0.02 \\
0.001 \\
0.001 \\
\text { n.S } \\
\text { n.S } \\
\text { n.S } \\
\text { n.S } \\
0.001 \\
\end{array}$ \\
\hline
\end{tabular}


Otherwise, the main mechanism that underlies pathologies such as atherosclerosis, diabetes, hypertension and vasculitis is due to endothelial dysfunction and the activation of prothrombotic phenomena. In fact, the presence of these pathologies causes liberation of free radicals and endothelial dysfunction. An excessive presence of free radicals, produced by molecular oxygen, if not compensated by the endogenous antioxidant system, creates cellular apoptosis. ${ }^{6}$

The inner ear, as a highly specialized organ for the functions of hearing and balance, is characterized by a high energy consumption metabolism that requires a regular and adequate supply of oxygen, guaranteed by a fine regulation of the cerebro-vascular flow, involving endothelium-dependent mechanisms mediated by the glycocalytic interface of the cochlear-vestibular microcirculation. ${ }^{6}$

Furthermore, patients with autoimmune thyroid diseases seem to have an elevated risk of tinnitus; in fact, tinnitus is more likely to occur in females with a history of thyroid disease. Nevertheless, there have been few studies on the pathophysiology of tinnitus in acquired hypothyroidism. ${ }^{20}$

Studies examining the effect of hypothyroidism on inner ear morphology have reported that the first inner ear structure shows morphologic changes in the tectorial membrane. A constant alteration in the normal structure was found in addition to thickening of the basilar membrane, as well as inner and outer hair cell degeneration, tectorial membrane irregularities, and debris in the cochlear duct. Studies examining the effect of hypothyroidism on cochlear function have suggested that abnormal thyroid hormone levels lead to defects in the neurological and morphological development of the organ of Corti, suggesting that thyroid hormones exert direct effect on the cochlea. ${ }^{21}$

The precise relationship between depression and tinnitus is not clear: tinnitus can predispose individuals to depression or can be an independent comorbidity in patients with depression. ${ }^{21}$

In many cross-sectional studies on chronic tinnitus, the presence of a psychiatric comorbidity has been demonstrated. Both depression and anxiety may be present as an independent disease or as a concomitant pathological condition, which suggests a review of the literature to shed light on the link between these diseases. ${ }^{22,23}$

Some authors propose a common neurobiological basis that identifies the limbic system, a structure responsible for some functions, including emotions and behavior, as a possible explanation. $^{22}$

An alternative theory suggests that tinnitus is related to psychological disorders through a mediator: cortisol. The existence of glucocorticoid receptors in the ear has been demonstrated, along with evidence that patients with tinnitus exhibit high levels of cortisol. ${ }^{23}$

In addition, drug interactions have unknown effects and may increase tinnitus, a drug-use counseling and a list of ototoxic drugs should be performed.

The ability of these drugs to influence the ion transport system and the ion channels and so influencing the ear and kidney ionic homeostasis could be a predicting factor for possible pharmaceutical-related ototoxicity. ${ }^{24}$

One of the objectives of our study was to verify the possible correlation between tinnitus and the chronic intake of some drugs. From our results only, proton pump inhibitors and levothyroxine showed a statistically significant correlation with the presence of chronic tinnitus.

The scientific literature concerning these associations is quite poor and contrasting and surely needs further investigations.

In a recent study, contrary to our results, it was supposed a possible favorable effect of PPI on inner ear disorders and tinnitus. The mechanisms supposed to act as protective factors are: i) the regulatory effect on inner ear homeostasis through the action of PPI on the gastric type of proton pump of the inner ear; ii) the influence on the vasomotor activity enabling better peripheral perfusion and oxygenation of the inner ear; iii) a possible anti-inflammatory peripheral effect. Anyway, this study did not show any significant result on this correlation. ${ }^{25}$

Contrariwise, a study on Gastroesophageal reflux disease (GERD), PPI treatment and hearing loss, underlined that GERD might be the cause of hearing loss. In fact, PPIs inhibit the hydrogen/potassium adenosine triphosphate enzyme system of gastric parietal cells, thereby inhibiting secretion of hydrogen ions into the gastric lumen. This enzyme is also expressed in the lateral wall of the cochlea and plays a critical role in the formation of the endocochlear potential. Thus, inhibition of this enzyme by PPI treatment could increase the risk of hearing loss. Anyway, from this study the use of PPI was not associated with hearing loss after accounting for GERD symptoms. ${ }^{26}$

Only an old study underlines the incidence of hearing impairment: tinnitus and vertigo correlated with the severity of hypothyroidism, ${ }^{27}$ while scientific evidence on the possible role of the levothyroxine on the genesis of tinnitus is missing.

Table 3. Univariate logistic regression.

$\begin{array}{lll}\text { Odds Ratio } & \text { P-value } & \text { Confidence interval }\end{array}$

\begin{tabular}{|c|c|c|c|}
\hline \multicolumn{4}{|l|}{ Associated disease } \\
\hline Neurological disorders & 6.12 & 0.02 & $1.33-28.08$ \\
\hline Depression & 7.39 & 0.001 & $2.95-18.49$ \\
\hline Anxiety & 3.29 & 0.001 & 1.9-5.77 \\
\hline TMJ disorders & 2.64 & 0.001 & $1.5-4.63$ \\
\hline Headache & 2.61 & 0.001 & $1.48-4.59$ \\
\hline Cardiovascular diseases & 0.72 & n.s & $0.33-1.56$ \\
\hline Hypertension & 1.84 & n.s & $0.99-3.4$ \\
\hline Diabetes & 1.97 & n.s & $0.76-5.12$ \\
\hline Dysthyroidism & 3.36 & 0.001 & $1.63-6.95$ \\
\hline \multicolumn{4}{|l|}{ Drugs } \\
\hline Levothyroxine & 2.98 & 0.008 & $1.32-6.33$ \\
\hline Proton-pump inhibitors & 2.91 & 0.021 & $1.17-7.23$ \\
\hline
\end{tabular}




\section{Conclusions}

In this study, we tried to evaluate the audiological characteristics in the subjects affected by chronic tinnitus in order to find a possible correlation with the comorbidities and any drugs intake, that can predict its progress. In our sample, men were more affected by chronic tinnitus than women, DPOAEs were more affected on the left side, and $60 \%$ of subjects had associated hearing loss. The side most affected by tinnitus was the left side (36\%), and the frequency most affected was $6000 \mathrm{~Hz}(45 \%)$ showing a significance for the left side $\mathrm{p}=0.021$ correlating with the intensity, with an average of $41.3 \mathrm{~dB}$. The duration of tinnitus was $>6$ months in 107 patients $(82.3 \%)$. The most represented type of tinnitus was the whistle type in 39 patients $(30 \%)$. In our group of patients with tinnitus, based on the total THI score, we found light, mild, moderate and severe preponderances with only 6 patients with a catastrophic degree.

We found that statistically significant comorbidities were: the presence of anxiety and depression, TMJ disorders, dysthyroidism, headache, levothyroxine and PPI intake.

Furthermore, our study had some limitations. First of all, in considering the comorbidities, the time of occurrence and the severity of the pathologies were not evaluated, nor was the dosage of the drugs taken by patients or the duration of the administrations assessed. Therefore, we believe that our observations have only an epidemiological relevance to be explored with further studies. For instance, the high prevalence of tinnitus, anxiety and depression suggests a review of the literature to shed light on the link between these diseases.

The mechanism of action of PPI could also intervene in the absorption mechanisms of other potentially ototoxic drugs, for example cardioaspirin, and therefore requires further investigation. Moreover, in considering the association between tinnitus and levothyroxine intake, the pathogenic mechanism could be related both to the underlying pathology and to the effect on the basal metabolism of the molecule and therefore also on the ionic pumps.

\section{References}

1. American National Standards Institute. Specification for audiometers. ANSI S3.6. New York: American National Standards Institute; 1969.

2. National Research Council (US). Committee on Hearing, Bioacoustics, and biomechanics. Tinnitus: Facts, Theories, and Treatments. Washington DC: National Academies Press (US); 1982.

3. Huang Y-S, Malcolm K, Hwang J-H. The association between tinnitus and the risk of ischemic cerebrovascular disease in young and middle-aged patients: A secondary case control analysis of a nationwide, population-based health claims database. PLoS One 2017;12:11.

4. Tunkel DE, Bauer CA, Granieri EC, et al. Clinical practice guideline: tinnitus. Otolaryngol Head Neck Surg 2014;151:S1-40.

5. Fuller TE, Haider HF, Kikidis D, et al. Different Teams, Same Conclusions? A Systematic Review of Existing Clinical Guidelines for the Assessment and Treatment of Tinnitus in Adults. Front Psychol 2017;8:206.

6. Chavez-Delgado ME, Vazquez-Granados I, Velasco-Rodriguez V. Cochleovestibular dysfunction in patients with diabetes mellitus, hypertension and dyslipidemia. Acta Otorrinolaringol Esp 2012;63:93-101.

7. Sullivan MD, Katon W, Harrop Griffiths J. Disabling tinnitus. Association with affective disorder. Gen Hosp Psychiatry
1988;10:285-91.

8. Zöger S, Svedlund J, Holgers KM. Relationship between tinnitus severity and psychiatric disorders. Psychosomatics 2006;47:282-8.

9. Langguth B, Hund V, Schecklmann M. Tinnitus Patients with Comorbid Headaches: The Influence of Headache Type and Laterality on Tinnitus Characteristics. Front Neurol 2017;8:440.

10. Lin BM, Curhan SG, Wang M, et al. Prospective Study of Gastroesophageal Reflux, Use of Proton Pump Inhibitors and H2-Receptor Antagonists, and Risk of Hearing Loss. Ear Hear 2017;38:21-7.

11. Vernon J, Griest S, Press L. Attributes of tinnitus that may predict temporomandibular joint dysfunction. Cranio 1992;10:282-8.

12. Committee on Hearing and Equilibrium guidelines for the evaluation of results of treatment of conductive hearing loss. AmericanAcademy of Otolaryngology-Head and Neck Surgery Ffoundation, Inc.Otolaryngol Head Neck Surg. 1995 Sep;113(3):186-7. https://www.ncbi.nlm.nih.gov/ pubmed/7675477

13. Aazh H, Moore BCJ. Factors Associated With Depression in Patients With Tinnitus and Hyperacusis. Am J Audiol 2017;5629.

14. Newmann CW, Spitzer JB. Development of Tinnitus Handicap Inventory. Arch Otolaryngol Head Neck Surg 1996;122:143-8.

15. Khalfa S, Dubal S, Jouvent R, et al. Psychometric normalization of a hyperacusis questionnaire. ORL J Otorhinolaryngol Relat Spec 2002;64:436-42.

16. Fioretti A, Tortorella F, Pavaci S. Validity of the Italian version of Khalfa's questionnaire on hyperacusis. Acta Otorhinolaryngol Ital 2015;35:110-5.

17. Kim HJ, Lee HJ, An SY, et al. Analysis of the prevalence and associated risk factors of tinnitus in adults. PLoS One 2015;10:5.

18. Bush FM. Tinnitus and otalgia in temporomandibular disorders. J Prosthet Dent 1987;58:495-8.

19. Massimo R, Antonio G, Giancarlo C. Somatosensory tinnitus: Current evidence and future perspectives. J Int Med Res 2017;45:933-47.

20. Mahafzah MT, Mahafza T, Hawari HH. Investigating the possible audiological effects of hypothyroidism. J Phonet Audiol 2018;4:1.

21. Geocze L, Mucci S, Penido Nde O. Systematic review on the evidences of an association between tinnitus and depression. Braz J Otorhinolaryngol 2013;79:106-11.

22. Wallhäusser-Franke E, Brade J, Seegmüller A, et al. Correction: Tinnitus: Distinguishing between Subjectively Perceived Loudness and Tinnitus-Related Distress. PLoS One 2012;7:9.

23. Wallhäusser-Franke E, Hörmann K, Repik I. Transition from Acute to Chronic Tinnitus: Predictors for the Development of Chronic Distressing Tinnitus. Front Neurol 2017;8:605.

24. Cianfrone G, Pentangelo D, Altissimi G. Pharmacological drugs inducing ototoxicity, vestibular symptoms and tinnitus: a reasoned and updated guide. Eur Rev Med Pharmacol Sci 2011;15:601-36.

25. Pirodda A, Raimondi MC, Borghi C. Tinnitus in patients on therapy with proton pump inhibitors (PPI) and in PPI non-users. Hear Balance Commun 2014;12:2:84-7.

26. Lin BM, Curhan SG, Curhan GC. Prospective study of gastroesophageal reflux, use of proton pump inhibitors and $\mathrm{H} 2-$ receptor antagonists and risk of hearing loss. Ear Hear 2017;38:21-7.

27. Bhatia PL, Gupta OP, Mishr SK. Audiological and vestibular function tests in hypothyroidism. Laryngoscope 1977;87:2082-9. 